\title{
Novel Detection and Decoding Technique to Optimize Performance in MIMO Communication Systems
}

\author{
Wurod Qasim Mohamed ${ }^{1}$ and Karim Hamidian ${ }^{2}$ \\ ${ }^{1}$ Department of Electronic Engineering, University of Diyala, Diyala, Iraq \\ Email: Wurod89@csu.fullerton.edu \\ ${ }^{2}$ Electrical Engineering Department, California State University Fullerton, CA, USA \\ Email:khamidian@fullerton.edu
}

Abstract- The fifth generation new radio (5G-NR) access networks are expected to provide very high capacity and ultra-reliability. We think these requirements could be met by using a large number of antenna elements at both ends of communication systems, and by transmitting, detecting and decoding the data signal in parallel fashion. Thus, we proposed an $N_{t} \times N_{r}$ MIMO communication system that is analyzed and improved, and expected to achieve both very high throughput and ultra-reliability simultaneously for millimeter waves. Such improvement is achieved through proposed novel detection and decoding technique termed as Parallel Processing and Decoding Algorithm. The proposed technique increases the signal processing speed and prevents error propagation, which may be present in linear serial decoding techniques. Simulation results verify and demonstrate the advantages of using the proposed parallel detection and decoding technique.

Keywords- MIMO, New Decoding.

\section{INTRODUCTION}

One of the major challenges for 4G- LTE and 5G New Radio (NR) is to maximize both the reliability and data rate of Multiple Input Multiple Output (MIMO) systems [19] [21]. The goal of the MIMO systems is to combat fading by exploiting the multipath propagations between multiple transmitting and receiving antenna elements through two unique techniques: spatial diversity and spatial multiplexing. Table 1 summarizes the MIMO categories [4].

TABLE 1. THE MIMO CATEGORIES

\begin{tabular}{|c|c|c|c|}
\hline MIMO technique & Purpose & Approach & Method \\
\hline Spatial diversity & $\begin{array}{c}\text { Improve } \\
\text { reliability }\end{array}$ & $\begin{array}{c}\text { Combat } \\
\text { multipath }\end{array}$ & Space time coding \\
\hline $\begin{array}{c}\text { Spatial } \\
\text { multiplexing }\end{array}$ & $\begin{array}{c}\text { Increase } \\
\text { capacity }\end{array}$ & $\begin{array}{c}\text { Exploit } \\
\text { multipath }\end{array}$ & $\begin{array}{c}\text { Spatial } \\
\text { de-multiplexing }\end{array}$ \\
\hline
\end{tabular}

Spatial diversity is a technique that improves the reliability of the transmission through transmitting multiple replicas of the same signal by using Space Time Coding (STC) method [4]. In other words, spatial diversity counters the effects of fading in the multipath channel.

Two common metrics that characterize the amount of spatial diversity are diversity order and diversity gain [4]. Diversity order represents the number of independent replicas of the same transmitted signal that are available at the receiver. In an $N_{t} \times N_{r}$ MIMO system, there are $N_{t} N_{r}$ possible propagation paths between the $N_{t}$ transmitting antennas and the $N_{r}$ receiving antennas. A general property of the MIMO communication systems that holds true for many modulation types in a Rayleigh fading channel is that the diversity order $\left(N_{d}\right)$ equals to the diversity gain $\left(G_{d}\right)$. That is, a MIMO system achieves full diversity if the equation of $N_{d}=G_{d}=N_{t} N_{r}$ is true. 
However, spatial multiplexing technique exploits multipath propagation to transmit the information at a rate up to channel capacity (channel capacity improvements) without increasing the required bandwidth by employing the spatial de-multiplexing method.

\section{MIMO COMMUNICATIONS TECHNIQUES}

As mentioned earlier, the two main classes of MIMO communication techniques are spatial diversity and spatial multiplexing, which are achieved by the space time coding and spatial de-multiplexing methods, respectively.

\section{A. Space Time Coding (STC)}

Space time coding technique is a coding method that enables transmission of replicas of a transmitted signal using two or more transmitting antennas to achieve full diversity. This results in increased transmission reliability of the MIMO system with lower computational complexity and CSIR only and without any additional bandwidth requirement. There are two primary types of space time codes; they are space time block codes (STBCs) and space time trellis codes (STTCs). STBCs can be further divided into two types: orthogonal space time block codes (OSTBCs) and non-orthogonal space time block code (NOSTBCs) introduced in [7].

In 1998, Alamouti coding was introduced in space time coding for MIMO communication systems [11]. Alamouti coding is a square OSTBC using two transmitting antennas and a single receiving antenna.

The Alamouti coding technique increases the consistency of the system and allows transmission of two symbols within two symbol periods without requiring the channel state information at the transmitter (CSIT). Alamouti coding can be generalized to include cases when the receiver has more than one antenna.

\section{B. Spatial Multiplexing (SM)}

The second major class of MIMO technique is spatial multiplexing. The key purpose of the spatial multiplexing is to increase the data transmission rate without consuming excess bandwidth by transmitting multiple independent data streams over multipath channels. Thus, this technique exploits the multipath propagation between the transmitter and the receiver.

In general, spatial multiplexing is obtained using layered space time (LST) coding, where a layer indicates a data stream from a single transmitting antenna [2], [12]. There are various types of LST coding and decoding methods for a spatial multiplexing technique; they are vertical, horizontal, and diagonal bell laboratory layered space time coding abbreviated as V-BLAST, H-BLAST, and D-BLAST, respectively. In addition to these methods, SM is achieved by combining it with space time coding resulting in either multigroup space time coding (MGSTCs) or through threaded space time coding (TSTC) method. All these coding methods assume that channel state knowledge is available at the receiver (CSIR) only. Also, there are four different types of decoding for these encoding methods. Each decoding method has its own advantages and complexities different from the others.

\section{OPTIMIZING PERFORMANCE OF MIMO COMMUNICATION SYSTEMS USING A UNIQUE DETECTION TECHNIQUE}


To enhance the performance of MIMO communication systems in Rayleigh fading environment, a new

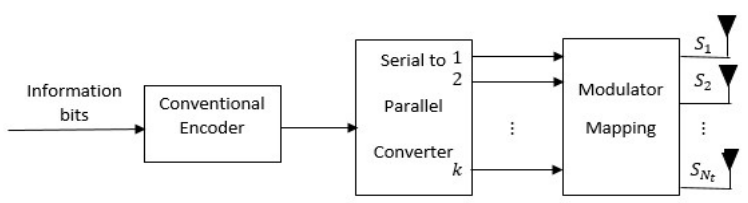

Fig. 1. MIMO Communication Transmitter Using V-BLAST.

proposed detection technique will be implemented to exploit the multipath propagation to decode $N_{t}$ received symbols simultaneously and independently [16]. At the transmitter, after channel encoder [6] the data stream splits into $N_{t}$ data streams, each of which is modulated separately and transmitted by its respective antenna as shown in fig. 1, [4].

This transmitter is implemented using V-BLAST encoding architecture and transmits $N_{t}$ modulated symbols over one symbol period. Therefore, the transmitted signal matrix $S$ is given as:

$S=\left[\begin{array}{llll}S_{1} S_{2} & \ldots & \ldots & S_{N_{t}}\end{array}\right]^{T}$

On the other hand, the receiver follows different strategies that enable the receiving system to detect and decode all received symbols in a parallel fashion as shown in Fig. 2.

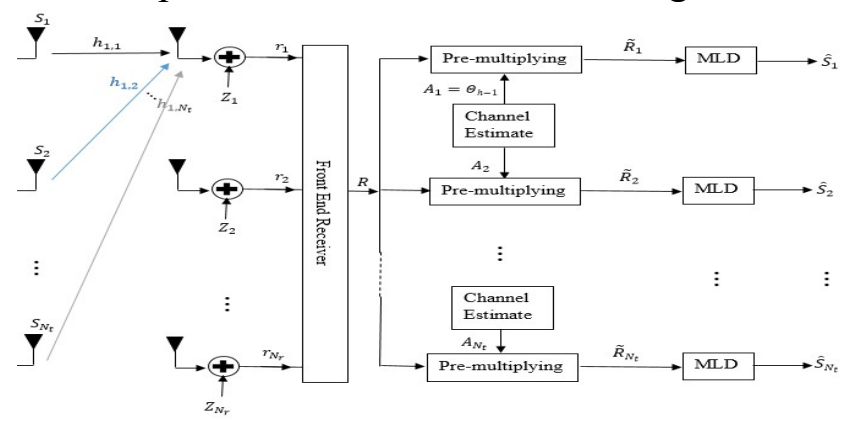

Fig.2. MIMO Communication Receiver Using Proposed Parallel Detection

That is, the received symbols will be processed simultaneously and independently. The channel matrix $H\left(N_{r} \times N_{t}\right)$ can be decomposed into components as shown in the following equation.

$H=\left[\begin{array}{llll}H_{1} & H_{2} & \ldots & H_{N_{t}}\end{array}\right]$

Where $H_{1}\left(N_{r} \times 1\right)$ refers to the channel component vector that is associated with the transmitted symbol $S_{1}$. Thus, the received interference that is associated with the transmitted symbol $S_{1}$, can be suppressed through truncating the resulting channel matrix $H_{h-1}\left(N_{r} \times\left(N_{t}-1\right)\right)$, where

$H_{h-1}=\left[\begin{array}{lll}H_{2} & \ldots & H_{N_{t}}\end{array}\right]$

To decode the symbol $\left(S_{1}\right)$ that is transmitted over the first transmitting antenna and to suppress all the remaining signals which constitute interference and that are associated with channel matrix $H_{h-1}$, it is necessary that the number of receiving antennas satisfies $N_{r} \geq N_{t}$. This suppression can be achieved through pre-multiplying the received signal matrix $R$ by the matrix $\Theta_{h-1}$ as shown in the equation below:

$$
\tilde{R}_{1} \triangleq \Theta_{h-1} R
$$

Where $\tilde{R}_{1}$ is estimate of the received signal after interference suppression, and $\Theta_{h-1}$ consists of the set of null space vector of $H_{h-1}\left(N_{r} \times\left(N_{t}-1\right)\right)$. The dimension of $\Theta_{h-1}$ is : 


$$
\operatorname{dim}\left[\Theta_{h-1}\right] \geq N_{r}-N_{t}+1
$$

Therefore, $\Theta_{h-1}$ is dimensioned as $\left[\left(N_{r}-N_{t}+1\right) \times N_{r}\right]$ and its rows are orthonormal vectors. This implies the following property:

$$
\Theta_{h-1} \Theta_{h-1}{ }^{H}=I_{\left(N_{r}-N_{t}+1\right)}
$$

Since $\Theta_{h-1}$ is the null space matrix of $H_{h-1}$ matrix, thus we have the following equation.

$$
\Theta_{h-1} H_{h-1}=0_{\left[\left(N_{r}-N_{t}+1\right) \times\left(N_{t}-1\right)\right]}
$$

Under the assumption of Rayleigh distribution channel, the received signal matrix is $R=\sqrt{\rho} H S+$ $Z$ [1], [4]. Using (2) and (7) into equation of $R$, it is easy to verify that $\tilde{R}_{1}$ from (4) can be express as:

$$
\tilde{R}_{1}=\sqrt{\rho} H_{1}{ }^{(e f f)} S_{1}+\bar{Z}_{1}
$$

Where $H_{1}{ }^{(e f f)}$ refers to the effective channel matrix that is associated with decoding of $S_{1}$ and represents the following.

$$
H_{1}{ }^{(e f f)}=\Theta_{h-1} H_{1}
$$

$\bar{Z}_{1}=\Theta_{h-1} Z$ is the noise term. It is interesting to note that (8) indicates that the energy of $\tilde{R}_{1}$ comes only from the $S_{1}$ symbol. This demonstrates that the interferences from all remaining transmitted symbols are nullified. In addition, there are $N_{r}$ replicas of symbol $S_{1}$ available at the receiver. This shows the proposed receiver achieves spatial diversity without using STC technique. We can decode symbol $S_{1}$ by applying the maximum likelihood decoding to (8). The result is shown in (10), where $\hat{S}_{1}$ refers to the estimate of $S_{1}$.

$$
\hat{S}_{1}=\arg \min _{\left\{S_{1}\right\}}\left\{\left\|\tilde{R}_{1}-\sqrt{\rho} H_{1}{ }^{(e f f)} S_{1}\right\|_{F}^{2}\right\}
$$

Simultaneously, during the detection of the first symbol, the receiver can detect and decode all the other received symbols through the following proposed parallel detection method. The decoding of the second transmitted symbol $S_{2}$ can be achieved through suppressing the interference that is associated with all the remaining transmitted symbols. This suppression is obtained by pre-multiplying the received matrix $R$ by $A_{2}$ matrix as follows.

$$
\tilde{R}_{2} \triangleq A_{2} R
$$

Where $\tilde{R}_{2}$ refers to the estimate of the received matrix after the interference suppression, and $A_{2}$ refers to the overall per-multiplying matrix, which represents the following.

$$
A_{2\left[\left(N_{r}-N_{t}+1\right) \times\left(N_{r}\right)\right]}=N\left[\Theta_{1} H_{h-1,2}\right] \Theta_{1}
$$

Where $\Theta_{1}$ refers to the null space of $\left[H_{1}\right]$, and $N\left[\Theta_{1} H_{h-1,2}\right]$ refers to the null space of $\left[\Theta_{1} H_{h-1,2}\right]$ with the following dimension $\left[\left(N_{r}-1\right) \times\left(N_{t}-2\right)\right]$.

Therefore, the dimension of $N\left[\Theta_{1} H_{h-1,2}\right]$ can be obtained by using the rank plus nullity theorem as follows.

$$
\operatorname{dim}\left[N\left[\Theta_{1} H_{h-1,2}\right]\right] \geq N_{r}-N_{t}+1
$$

Thus, the dimension of $N\left[\Theta_{1} H_{h-1,2}\right]$ is $\left[\left(N_{r}-N_{t}+1\right) \times\left(N_{r}-1\right)\right]$. Assuming Rayleigh flat fading channel, the received signal matrix is $R=\sqrt{\rho} H S+Z$,[2], [4]. Using (2), (12) and equation of $R$ into equation (11), the resulting $\tilde{R}_{2}$ matrix can be expressed as follows.

$$
\tilde{R}_{2}=\sqrt{\rho} H_{2}{ }^{(e f f)} S_{2}+\bar{Z}_{2}
$$

Where $\mathrm{H}_{2}{ }^{(e f f)}$ refers to the effective channel matrix that is associated with decoding of $S_{2}$ and it is defined by (15).

The noise term associated with receiving of $S_{2}$ is $\bar{Z}_{2}=A_{2} Z$.

$$
H_{2}{ }^{(e f f)}=A_{2} H_{2}
$$




\section{Gyancity Journal of Engineering and Technology, Vol.5, No.1, pp. 34-46, January 2019 ISSN: 2456-0065 DOI: 10.21058/gjet.2019.51005}

It is clear from (14) that the energy of $\tilde{R}_{2}$ is only from symbol $S_{2}$. The estimate of the received symbol $S_{2}$ can be obtained by applying the maximum likelihood decoding algorithm to (14) as shown in (16), where $\hat{S}_{2}$ refers to the estimate of $S_{2}$.

$$
\hat{S}_{2}=\arg \min _{\left\{S_{2}\right\}}\left\{\left\|\tilde{R}_{2}-\sqrt{\rho} H_{2}{ }^{(e f f)} S_{2}\right\|_{F}^{2}\right\}
$$

The detection and decoding of the remaining symbols $S_{3} \ldots . S_{N_{t}}$ are similar to the previous processing steps. In general, the decoding of $S_{i}$ transmitted symbol is achieved through the following premultiplying operation.

$$
\tilde{R}_{i}=A_{i} R
$$

Where $\widetilde{R}_{i}$ refers to the estimate of the received matrix after the interference suppression, and $A_{i}$ refers to the overall per-multiplying matrix, which can be expressed as shown in (18).

$A_{i\left[\left(N_{r}-N_{t}+1\right) \times\left(N_{r}\right)\right]}=$

$$
N\left[\Theta_{1+2+. .+(i-1)} H_{h-1,2, . .,}\right] \Theta_{1+2+. .+(i-1)}
$$

Where $\Theta_{1+2+. .+(i-1)}$ refers to the null space of $\left[H_{1+2+. .+(i-1)}\right]$, which itself represents the following.

$$
H_{1+2+. .+(i-1)}=\left[\begin{array}{llll}
H_{1} & H_{2} & \ldots & H_{i-1}
\end{array}\right]_{\left[\left(N_{r}\right) \times\left(\sum_{j=1}^{i-1} 1\right)\right]}
$$

Where, $N\left[\Theta_{1+2+. .+(i-1)} H_{h-1,2, . .,}\right]$ refers to the null space of $\left[\Theta_{1+2+. .+(i-1)} H_{h-1,2, . ., i}\right]$ with dimension $\left[\left(N_{r}-\left(\sum_{j=1}^{i-1} 1\right)\right) \times\left(N_{t}-\left(\sum_{j=1}^{i} 1\right)\right)\right]$

Therefore, the dimension of $N\left[\Theta_{1+2+. .+(i-1)} H_{h-1,2, . .,}\right]$ using the rank plus nullity theorem must satisfy equation (20)

$$
\operatorname{dim}\left[N\left[\Theta_{1+2+. .+(i-1)} H_{h-1,2, . .,}\right]\right] \geq N_{r}-N_{t}+1
$$

Consequently, the dimension of $N\left[\Theta_{1+2+. .+(i-1)} H_{h-1,2, . ., i}\right]$ is $\left[\left(N_{r}-N_{t}+1\right) \times\left(N_{r}-\left(\sum_{j=1}^{i-1} 1\right)\right)\right]$. Thus, the dimension of $A_{i}$ is $\left[\left(N_{r}-N_{t}+1\right) \times\left(N_{r}\right)\right]$ as shown in equation (18).

Therefore, the estimate of the received signal matrix $\tilde{R}_{i}$ can be obtained using similar steps to those used to estimate $\tilde{R}_{2}$.

$$
\tilde{R}_{i}=\sqrt{\rho} H_{i}^{(e f f)} S_{i}+\bar{Z}_{i}
$$

Where $H_{i}{ }^{(e f f)}$ refers to the effective channel matrix that is associated with decoding of symbol $S_{i}$ and its expression is shown in the following equation.

$$
H_{i}^{(e f f)}=A_{i} H_{i}
$$

The noise term that is associated with receiving $S_{i}$ is $\bar{Z}_{i}=A_{i} Z$. The estimate $\hat{S}_{i}$ of the received symbol $S_{i}$ is obtained by applying maximum likelihood decoding to (21) as follows.

$$
\hat{S}_{i}=\arg \min _{\left\{S_{i}\right\}}\left\{\left\|\tilde{R}_{i}-\sqrt{\rho} H_{i}^{(e f f)} S_{i}\right\|_{F}^{2}\right\}
$$

In summary, the transmitter sends $N_{t}$ modulated symbols over one symbol period using spatial multiplexing technique, and the receiver is enabled to detect and decode all these symbols simultaneously and independently by using the proposed decoding algorithm as outlined above. This suggests that the speed of signal processing at the receiver, and thus the throughput, will be significantly increased. In addition, at any given symbol period, this algorithm enables the receiver to have access to twice of $\left(N_{r}-N_{t}+1\right)$ replicas of each transmitted symbol. As a result, the reliability of the system is improved simultaneously. 


\section{Gyancity Journal of Engineering and Technology, Vol.5, No.1, pp. 34-46, January 2019 ISSN: 2456-0065 DOI: 10.21058/gjet.2019.51005}

Hence, it is important to note that this system achieves both spatial diversity and spatial multiplexing simultaneously without using STC technique. However, MGSTCs method also achieves both spatial diversity

and spatial multiplexing simultaneously, but there are three major differences between these two methods. First, MGSTCs method requires that the number of transmitting antenna to satisfy $N_{t} \geq 4$, while the proposed algorithm requires that $N_{t} \geq 2$. The second improvement is that this novel algorithm significantly improves the signal processing speed at the receiver. In standard MGSTCs method, the decoding of the component codes is performed iteratively and in a serial fashion. While using the new algorithm, the decoding of component codes are performed simultaneously and in a parallel way. Furthermore, the proposed parallel processing technique improves the probability of bit error (BER) by preventing 'possible error propagation. In standard MGSTCs method, the decoding of current component code symbol depends on the values of all previously decoded component codes. This indicates that if an error occurs in decoding of

a component code symbol, then such error will propagate to all remaining component codes. This problem is resolved in the proposed decoding algorithm since the receiver decodes the symbols independently and simultaneously. Fig.3 summarizes the new parallel decoding algorithm.

In general, the diversity order depends mostly on the difference between the numbers of receiving and

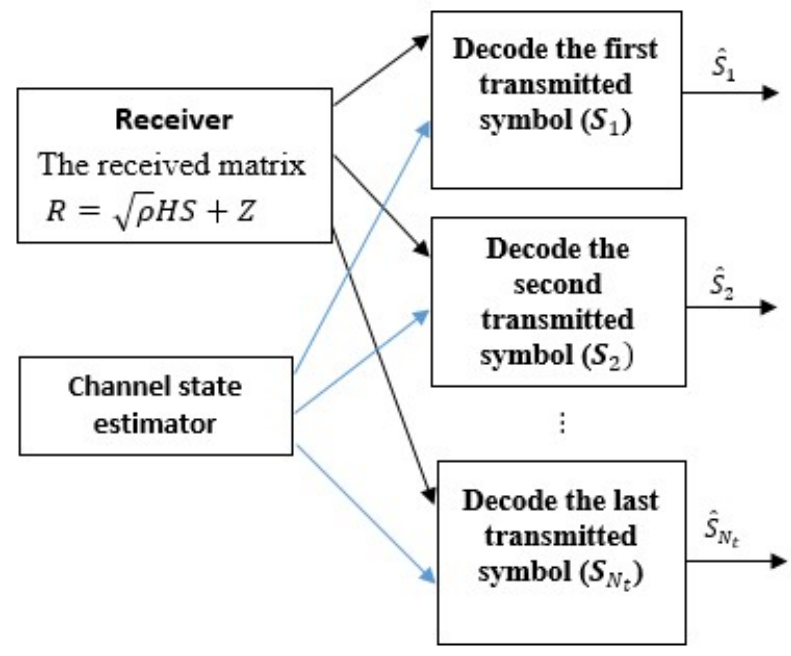
transmitting antennas. This difference also increases the gain of the replicas of each symbol. Like so, the diversity order depends on the number of the rows of the premultiplying matrix $\left(N_{r}-N_{t}+1\right)$ and the diversity order $\left(N_{d}\right)$ of MIMO communication systems using this parallel decoding technique is:

$$
\begin{array}{r}
N_{d}=2 *\left(N_{r}-N_{t}\right. \\
+1)
\end{array}
$$

Also, for $\left(\mathrm{N}_{\mathrm{t}} \times \mathrm{N}_{\mathrm{r}}\right)$ MIMO communication systems, the channel capacity is given by the expression shown in (25) [2] , [3] and [4].

$$
\begin{aligned}
C_{\left(\mathrm{N}_{\mathrm{t}} \times \mathrm{N}_{\mathrm{r}}\right) M I M O} & =\sum_{i=1}^{\mathrm{r}} \log _{2}(1 \\
& \left.+\frac{\rho}{\mathrm{N}_{\mathrm{t}}} \lambda_{i}\right)
\end{aligned}
$$

Decoding Algorithm.

Where $\lambda_{i}$ is the eigenvalue of the channel matrix, and $r$ is the rank of the channel matrix.

\section{SIMULATION RESULTS}

In this section, we assume that $N_{t}=2$ and $N_{r}=2,3$, and 4 respectively, and that the transmitter employs spatial multiplexing technique. The receiver can extract two

transmitted symbols independently and simultaneously by using the new decoding algorithm [16]. Fig. 4 shows the performance results of the proposed decoding method for a $(2 \times 2)$ MIMO communication system compared with two similar systems; one using Alamouti coding technique that can achieve only spatial diversity, and the other employs spatial multiplexing that uses serial decoding and can achieve only spatial multiplexing. In figure 4 , the BER is plotted against $E_{b} / N_{0}$ in $\mathrm{dB}$ in a Rayleigh fading channel and using 


\section{Gyancity Journal of Engineering and Technology, Vol.5, No.1, pp. 34-46, January 2019 ISSN: 2456-0065 DOI: 10.21058/gjet.2019.51005}

BPSK modulation for different systems. Our proposed decoding method is unique because currently there are no $2 \times 2$ MIMO system implemented using Alamouti coding algorithm that can achieve both spatial diversity and spatial multiplexing simultaneously. The bit error probability expression of parallel decoding system is

$$
\begin{array}{r}
P_{b(2 \times 2) M I M O-P a r a l l e l ~ d e c o d i n g} \\
=\left[\frac{1}{2}(1-\mu)\right]^{2}(2+\mu)(26)
\end{array}
$$

Where $\mu=\sqrt{\frac{\bar{\rho}}{1+\bar{\rho}}}$.

Where $\bar{\rho}$ refers to the time averaged signal-to-noise ratio.

At regions where the diversity order represents the slope of the bit error probability curve, the diversity order of the system approaches to $N_{d}=2$. The results show that the system using the new decoding algorithm, achieves considerably lower bit errors rate (BER) than

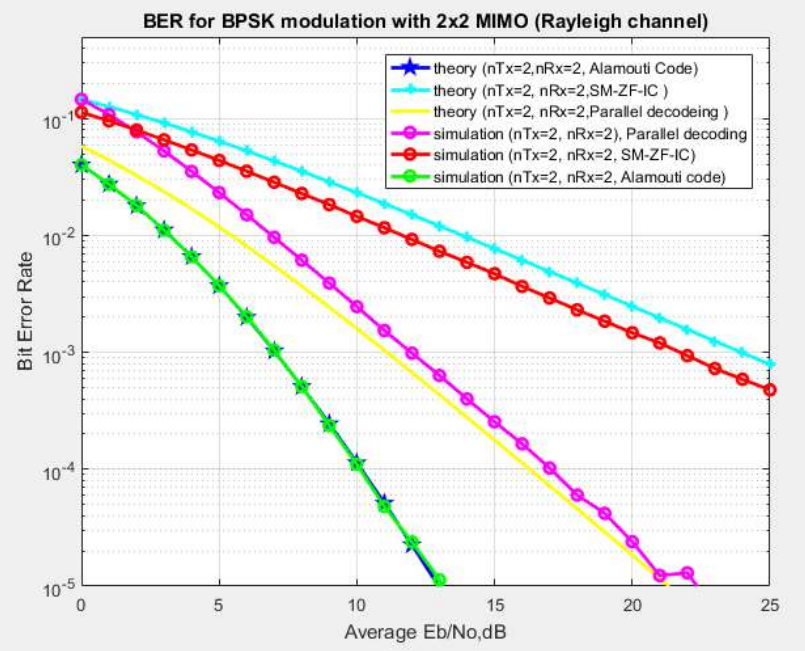

Figure 4. Comparison of BER for different types 2X2 MIMO systems

the system that only uses a spatial multiplexing technique. Nonetheless, the new model has a higher bit errors rate than the system that uses Alamouti coding technique only.

On the other hand, the proposed parallel processing algorithm improves the channel capacity as shown in Fig. 5. The figure shows that the channel capacity of the proposed system is equal to the channel capacity of a $2 \times 2$ MIMO system that uses spatial multiplexing technique, and it is twice of the channel capacity of a $2 \times 2$ MIMO system that uses Alamouti code.

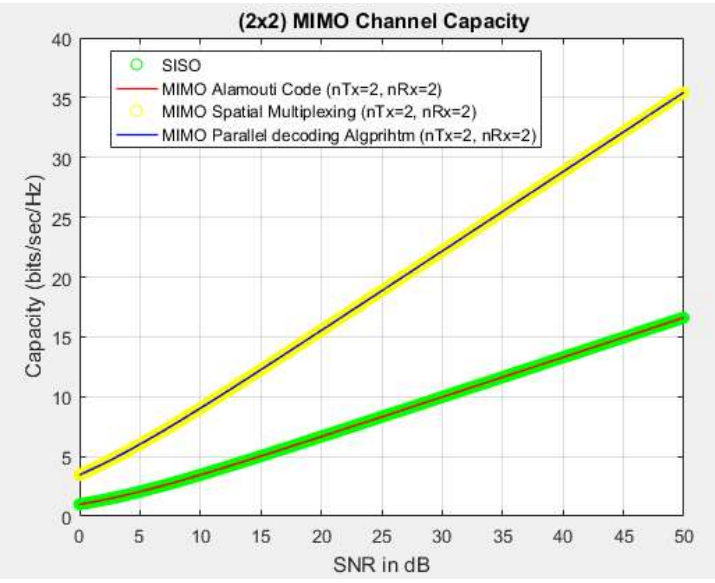

Fig. 5. Channel capacity comparison of different $2 \times 2$ MIMO systems.

Increasing the number of the receiving antennas leads to more improvements in the performance of MIMO communications systems that use the proposed detection algorithm. Fig. 6 shows the performance results of $(2 \times 3)$ MIMO communications system using the proposed model compared with a $(2 \times 3)$ MIMO system using only Alamouti code and a $(2 \times 3)$ MIMO system using only spatial multiplexing and 
serial decoding in a Rayleigh fading channel under the assumption of using BPSK modulation. The theoretical expression of the bit error probability of these methods is given in (27).

$P_{b(2 \times 3) M I M O-P a r a l l e l}$ decoding $=$

$$
\left[\frac{1}{2}(1-\mu)\right]^{4} \sum_{k=0}^{3}\left(\begin{array}{c}
3+k \\
k
\end{array}\right)\left[\frac{1}{2}(1+\mu)\right]^{k}
$$

The results show the diversity order of this system approaches to $N_{d}=4$.

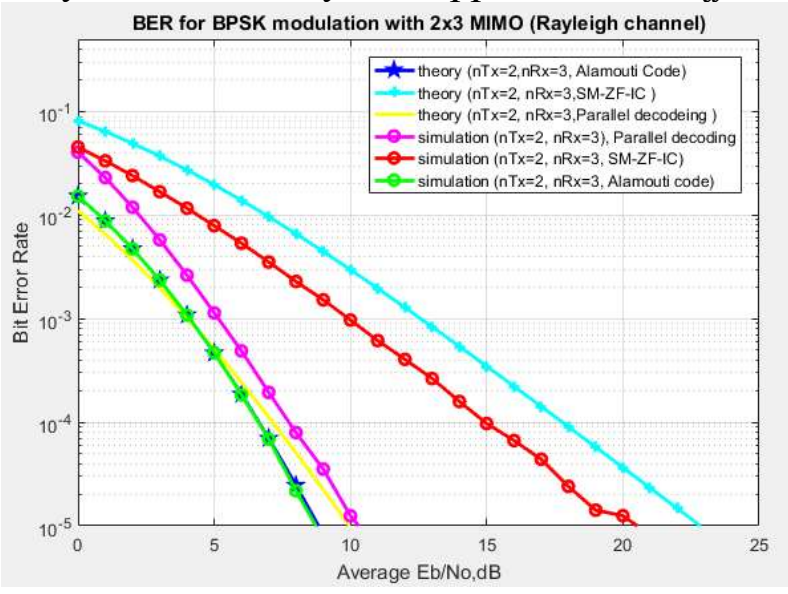

Fig. 6. BER for different types of $2 \times 3$ MIMO systems.

Comparing the performance of different types of $(2 \times 3)$ MIMO systems, the results show that the system using the parallel decoding algorithm achieves significantly lower BER than the system that uses spatial multiplexing technique only. However, it is very close to the BER of the system that only uses Alamouti coding technique. On the other hand, the proposed decoding algorithm improves the channel capacity as shown in Fig. 7. In the figure, the channel capacity of our model is equal to the channel capacity of the system using spatial multiplexing technique, and it is more than twice the channel capacity of the system using Alamouti coding technique.

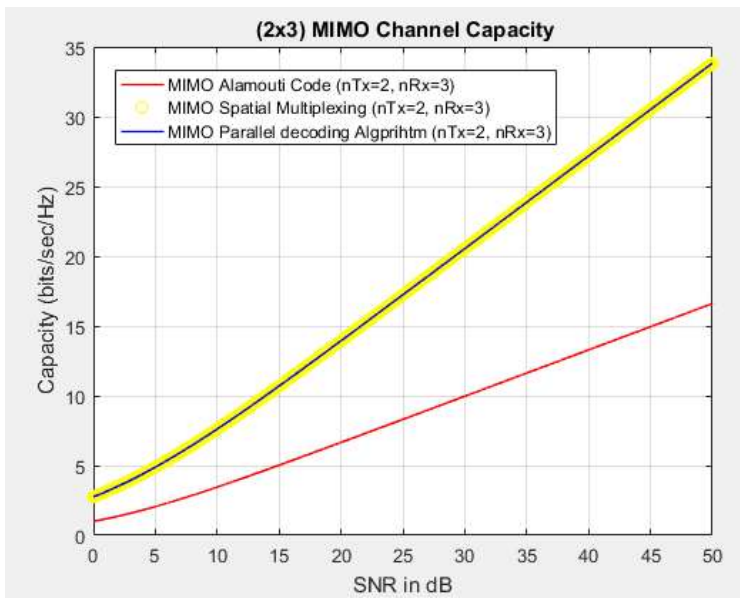

Fig. 7. Channel capacity comparison for $2 \times 3$ MIMO systems 


\section{Gyancity Journal of Engineering and Technology, \\ Vol.5, No.1, pp. 34-46, January 2019 \\ ISSN: 2456-0065 DOI: 10.21058/gjet.2019.51005}

Finally, fig. 8 shows the performance results of a $(2 \times 4)$ MIMO communication system using the new decoding model compared to a $(2 \times 4)$ MIMO system using Alamouti code and a $(2 \times 4)$ MIMO system using spatial multiplexing in a Rayleigh fading channel under assumption of using BPSK modulation. The theoretical expression of the bit error probability for these methods is

$P_{b,(2 \times 4) M I M O-P a r a l l e l}$ decoding $=$

$$
\left[\frac{1}{2}(1-\mu)\right]^{6} \sum_{k=0}^{5}\left(\begin{array}{c}
5+k \\
k
\end{array}\right)\left[\frac{1}{2}(1+\mu)\right]^{k}
$$

The results show the diversity order of this system approaches to $N_{d}=6$.

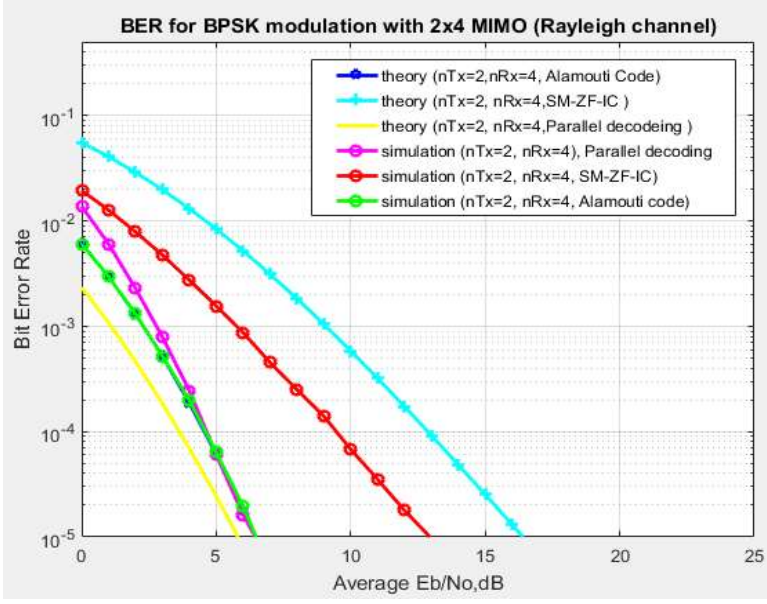

Fig. 8. BER of different types of $2 \times 4$ MIMO systems.

The results indicate that the system that uses the proposed decoding algorithm achieves significantly lower bit errors than the system that uses spatial multiplexing technique only, but its BER of our model becomes more close to the BER of the system that uses only Alamouti coding technique. This is due to increasing the difference between the number of receiving and transmitting antennas. On the other hand, the parallel decoding algorithm improves the channel capacity. As shown in the figure 9, the channel capacity of the system using the new decoding algorithm is equal to the channel capacity of the system using spatial multiplexing technique, and it is more than twice the channel capacity of a similar system using Alamouti coding technique.

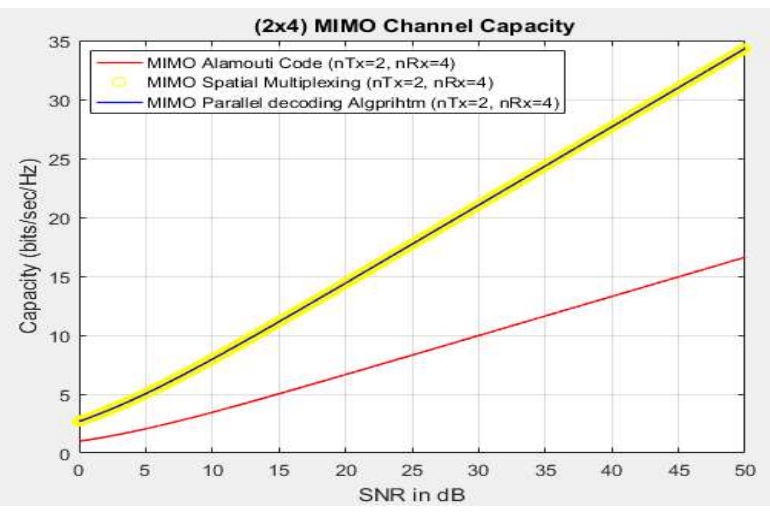

Fig. 9. A $2 \times 4$ MIMO channel capacity comparison. 


\section{Gyancity Journal of Engineering and Technology, Vol.5, No.1, pp. 34-46, January 2019 ISSN: 2456-0065 DOI: 10.21058/gjet.2019.51005}

From figures 8 and 9, we see the that system which uses the parallel decoding algorithm and employs a spatial multiplexing technique improves the throughput and achieves a significantly better diversity order, which matches closely with the diversity order of a similar system using Alamouti coding technique.

In our model, increasing the number of receiving antennas significantly improves the reliability of $2 \times N_{r}$ MIMO communication systems. More precisely, increasing the difference between the number of receiving and transmitting antennas improves the BER and in turn, the reliability of the system. Fig. 10 shows these improvements.

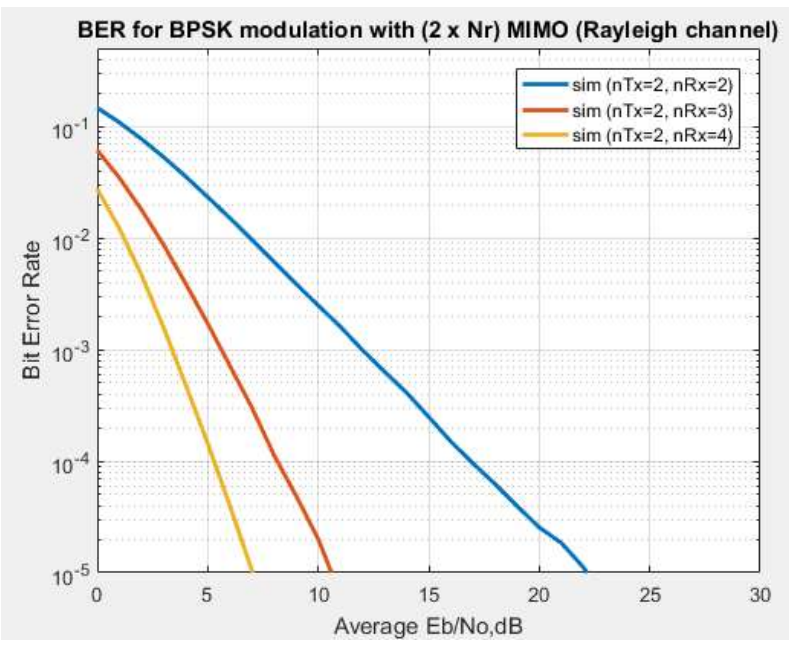

Figure 10. Bit error probability of $2 \times \mathrm{N}_{\mathrm{r}}$ MIMO communication systems.

The results show that the probability of bit error of a $2 \times 4$ MIMO communication system is significantly smaller than BER of any of the other $2 \times N_{r}$ MIMO communication systems with $2 \leq N_{r} \leq$ 3, which use the parallel decoding technique.

On the other hand, it should be noted that increasing the number of the receiving antennas with a fixed number of the transmit antennas, slightly enhances the throughput of the system as shown in Fig. 11. This occurs due to the different dimensions of the channel matrix, which itself implies increasing the number of the eigenvalues of the channel matrix.

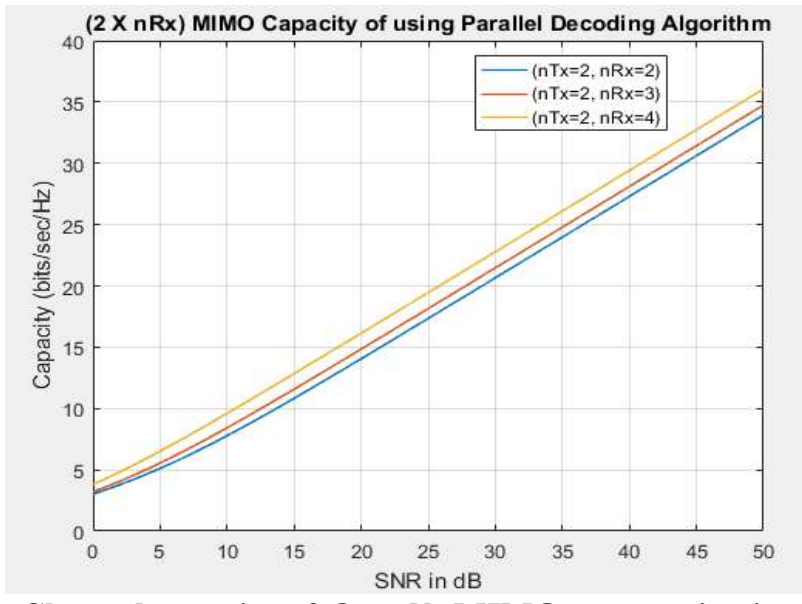

Fig. 11. Channel capacity of $2 \times \mathrm{N}_{\mathrm{r}}$ MIMO communication systems. 
The results show that the channel capacity of a $2 \times 4$ MIMO communication system is slightly more than any of the other $2 \times N_{r}$ MIMO communication systems using the new detection method. Table 2 summarizes the performance results of $2 \times N_{r}$ MIMO systems using the novel technique.

TABLE 2. PERFORMANCE COMPARISON OF $2 \times 2$ MIMO, $2 \times 3$ MIMO, AND $2 \times 4$ MIMO COMMUNICATION SYSTEMS [20]

\begin{tabular}{|l|c|c|c|l|}
\hline \multirow{2}{*}{ Parameter } & \multicolumn{3}{|c|}{ System } & \multirow{2}{*}{ Remarks } \\
\cline { 2 - 5 } Channel capacity & $\begin{array}{l}2 \times 2 \mathrm{MIMO} \\
\begin{array}{l}C_{(2 \times 2) M I} \\
=2 * C_{S I S O}\end{array}\end{array}$ & $\begin{array}{l}C_{(2 \times 3) M I M O} \\
\geq 2 * C_{S I S O} \\
\text { due to the } \\
\text { eigenvalues of }(2 \times \\
\text { 3) channel matrix }\end{array}$ & $\begin{array}{l}C_{(2 \times 4) M I M O} \\
\geq 2 * C_{S I S O} \\
\text { due to the } \\
\text { eigenvalues of }(2 \times \\
4) \text { channel matrix }\end{array}$ & $\begin{array}{l}2 \times 4 \text { MIMO } \\
\text { Capacity is } \\
\text { better than both } \\
\text { the other } \\
\text { systems }\end{array}$ \\
\hline Diversity order & $N_{d}=2$ & $N_{d}=4$ & $\begin{array}{l}\text { Diversity order } \\
\text { of a } 2 \times 4 \\
\text { MIMO is the } \\
\text { best }\end{array}$ \\
\hline
\end{tabular}

Usually, the system that employs a spatial multiplexing technique at the transmitter and applies the parallel decoding algorithm at the receiver improves both, system throughput and reliability simultaneously, which cannot be achieved by a similar system that uses serial and iterative decoding techniques.

Fig. 12 summarizes the improvements of $2 \times N_{r}$ MIMO communication systems that use the parallel decoding technique compared to corresponding systems that use only spatial multiplexing technique. The figure illustrates that at higher value of signal to noise ratio, the difference between bit error rates significantly increases when the number of receiving antennas are increased. This implies that the performance of the parallel decoding technique is better in terms of BER.

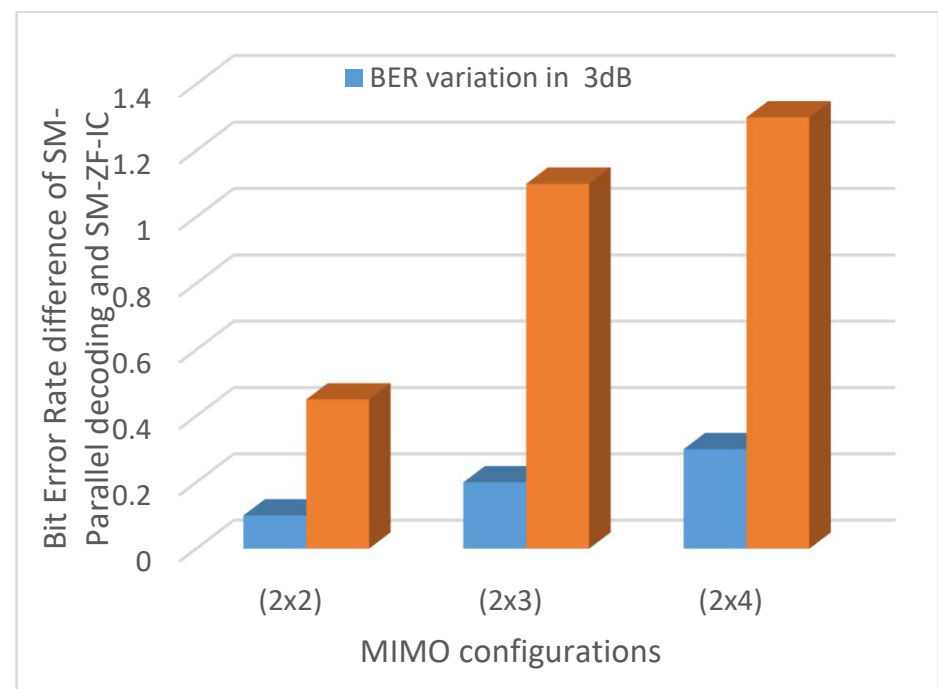

Fig. 12. Bit error rate difference of using parallel decoding and spatial multiplexing techniques for various $2 \times \mathrm{N}_{\mathrm{r}}$ MIMO communication systems. 


\section{Gyancity Journal of Engineering and Technology, Vol.5, No.1, pp. 34-46, January 2019 ISSN: 2456-0065 DOI: 10.21058/gjet.2019.51005}

Fig. 13 illustrates the improvements of $2 \times N_{r}$ MIMO communication systems that use the parallel decoding technique compared with corresponding systems that only use Alamouti code technique. The difference between bit error rates significantly decreases while increasing the number of the receiving antennas for higher value of signal to noise ratio. This implies that the performance achieved using parallel decoding method approaches the performance of the Alamouti coding technique when the signal to noise ratio is sufficiently large. As shown in the previous figures, the proposed parallel decoding technique provides optimum performance results with a non-square channel matrix.

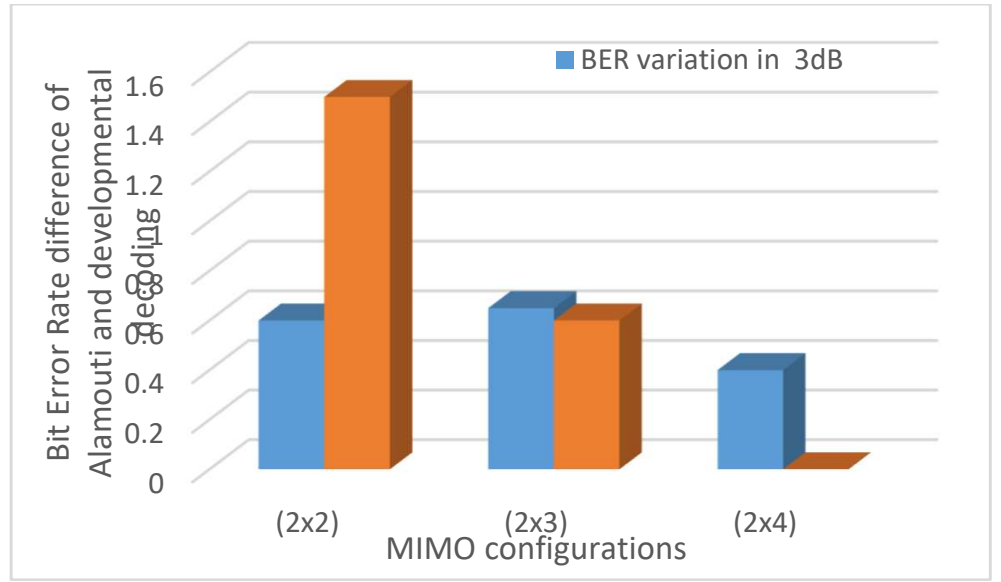

Fig. 13. Bit error rate difference of using parallel decoding and Alamouti code techniques for various $2 \times \mathrm{N}_{\mathrm{r}}$ MIMO communication systems.

\section{CONCLUSION}

The 5G- NR access networks are expected to provide very high capacity and ultra-reliability. To achieve these requirements, we focused on the study and investigation of MIMO communication techniques that use large number of antenna elements (antenna array) at both end of communication systems and developed novels ways to enhance the overall performance of $\left(N_{t} \times N_{r}\right)$ MIMO systems. A new detection and decoding technique called parallel detection and decoding algorithm was developed and applied to MIMO communication systems. The simulation and performance results confirm the improvements offered by the new parallel decoding method. It was shown that the new technique increases the throughput of the system when compared to similar systems that use iterative and serial detection method. In addition, it was observed that the proposed method prevents error propagation by extracting all received symbols independently and simultaneously. Moreover, it was shown that parallel decoding technique reduces the bit error probability and increases the speed of signal transmission without using any STC techniques. However, it was seen that the BER of the proposed algorithm is slightly higher than a similar system using STC technique such as Alamouti code. The BER performance of these two techniques become closer as $N_{r}$ assumes value of 4 or higher in the $2 \times N_{r}$ MIMO system.

Finally, it was seen that with the new decoding method, the bit error probability, BER, decreases significantly when the difference between the number of receiving and transmitting antennas is increased.

\section{REFERENCES}

[1] Bernard Sklar. Digital Communications Fundamentals and Applications, $2^{\text {nd }}$ edition. Prentice Hall P T R. 


\section{Gyancity Journal of Engineering and Technology, Vol.5, No.1, pp. 34-46, January 2019 ISSN: 2456-0065 DOI: 10.21058/gjet.2019.51005}

[2] B. P. Lathi and Zhi Ding. Modern Digital and Analog Communication Systems, 4th edition. Oxford University Press, Inc. 2009.

[3] Durgin Gregory. D. Space-Time Wireless Channels. Prentice Hall PTR, NJ 07458: Pearson Education. Inc., 2003.

[4] Hampton Jerry R. Introduction to MIMO Communications. New York: Cambridge University Press, 2014.

[5] Hamidian. Karim. Introduction to Cellular Wireless Communication. San Diego, CA: Montezuma Publishing, 2015.

[6] Hamidian Karim. Information Theory and Coding. San Diego, CA: Montezuma Publishing, 2014.

[7] Hamid Jafarkhani. Space-Time Coding: Theory and Practice. Cambridge University Press, 2010.

[8] John Proakis and Masoud Salehi. Digital Communications, 5th edition. McGraw Hill Science/Engineering/Math, 2007.

[9] P.F. Driessen and G.J. Foschini. On the capacity formula for multiple input-multiple output wireless channels: a geometric interpretation. 1999 IEEE International Conference on, Communications, ICC '99. 3:1603-1607, 1999.

[10] Sahu, A. K., \& Singh, S. S. (2012, October/November). BER Performance Improvement Using MIMO Technique Over Rayleigh Wireless Channel with Different Equalizers. International Journal of Engineering and Technology (IJET), 4, 5th ser., 333-340.

[11] S.M. Alamouti. A simple transmit diversity technique for wireless commun-ications. IEEE Journal on Selected Areas in Communications, 16(8):1451-1458, October 1998.

[12] Toshio, M., Tomoyuki, O., Hitoshi, Y., \& Narumi, U. The Overview of the 4th Generation Mobile Communication System. IEEE, (2005). PP.1551-1555.

[13] Theodore S. Rappaport. Wireless Communications: Principles and Prac-tice, 2nd edition. Prentice Hall, 2002.

[14] V. Tarokh, A. Naguib, N. Seshadri, and A.R. Calderbank. Combined array processing and space-time coding. IEEE Transactions on Information Theory, 45(4):1121-1128, May 1999.

[15] Yong, C. S., Jaekwon, K., Won, Y. Y., \& Chung, K. G. MIMO-OFDM Wireless Communications with MATLAB. John Wiley \& Sons (Asia) Pte., 2010.

[16] Mohamed Wurod Q., Performance Analysis of a New Decoding Technique For MIMO And MIMO - OFDM Communication Systems, MS thesis, Fall 2016 California State University, Fullerton.

[17] Hamidian. Karim, and Mohamed Wurod Q., Performance Enhancement of MIMO - MGSTC using a New Detection and Decoding Technique,IEEE, Future of Information and Communication Conference (FICC) 2018, April 2018, Singapore

[18] Eko Onggosanusi,Md Saifur, et al, IEEE Communication Magazine March 2018

[19] 3GPP TR 38.802 v14.1.0, "Study on New Radio Access Technology, Physical Layer Aspects."

[20] 3GPP TR 38.913 v14.3.0, "Study on Scenarios and Requirements for Next Generation Access Technologies".

[21] NGMN Alliance, "NGMN 5G White Paper," Feb. 2015; https://www.ngmn.org/fileadmin/ngmn/content/downloads/Technical/2015/NGMN_5G_White_Paper_V1_0.pd f, accessed 7 Feb. 2018

[22] 3GPP TR 38.802 v14.2.0, "Study on New Radio Access Technology Physical Layer Aspects (Release 14)," Sept. 2017

[23] Jin Liu, Kelvin Au, et al, IEEE Communication Magazine March 2018 